\title{
Screening probiotics from Lactobacillus strains according to their abilities to inhibit pathogen adhesion and induction of pro-inflammatory cytokine IL-8
}

\author{
Yanfeng Tuo,, Xing Song, , Yinglong Song, ${ }^{*}$ Wenwen Liu, ${ }^{*}$ Yu Tang, ${ }^{*}$ Yuan Gao, ${ }^{*}$ Shujuan Jiang, ${ }^{*}$ Fang Qian, ${ }^{*}$ \\ and Guangqing $\mathrm{Mu}^{*} \boldsymbol{\dagger}^{1}$ \\ *School of Food Science and Technology, Dalian Polytechnic University, Dalian, 116034, P. R. China \\ †Synergetic Innovation Center of Food Safety and Nutrition, Northeast Agricultural University, Harbin, 150030, P. R. China
}

\begin{abstract}
Probiotics can be screened according to their abilities to inhibit pathogen adhesion and inhibit the production of pro-inflammatory cytokines. Eleven Lactobacillus strains isolated from traditional fermented dairy foods in Xinjiang, China, were studied for their potential to inhibit adhesion of Escherichia coli to intestinal epithelial cells and to inhibit E. coli-induced production of interleukin (IL)-8 by intestinal epithelial cells. The results showed that the 11 strains could inhibit adhesion of E. coli to Caco-2 cell monolayers and inhibit the induction of IL-8 production by $E$. coli in HT-29 cells. The inhibiting activities of the Lactobacillus strains against E. coli adhesion and IL-8 induction were strainspecific and not positively correlated, whereas the excluding activity of the strains against $E$. coli adhesion and their coaggregation with E. coli were positively correlated. The effector molecules of the strains with probiotic potential should be identified to explain the mechanism behind these observations.
\end{abstract}

Key words: Lactobacillus, adhesion, inflammatory, IL-8

\section{INTRODUCTION}

Probiotics are live microorganisms that, when consumed in adequate amounts, produce beneficial effects on host, including restoring gastrointestinal tract flora, immunomodulation, and so on (FAO/WHO, 2001; Fukuda et al., 2011; Sánchez and Urdaci, 2012; Unno et al., 2015).

Specific probiotics should be selected based on their ability to inhibit adhesion or displace pathogens adhering to human intestinal mucosa (Collado et al., 2007). Adhesion and colonization of pathogenic bacteria on

Received August 9, 2017.

Accepted September 4, 2017.

${ }^{1}$ Corresponding author: gq6552002@aliyun.com the intestinal mucosa are important steps in pathogenic infection. Probiotics are able to adhere to the intestinal mucosa and thus inhibit the binding of pathogens and invasion of intestinal epithelial cells (Faghfoori et al., 2015). As probiotic strains, some Lactobacillus strains are reported to adhere to intestinal epithelium, inhibiting invasion by pathogens and improving epithelial barrier function (Chen et al., 2012; Saxami et al., 2016; Yu et al., 2017).

Immunomodulation capacity of Lactobacillus strains is considered a criterion for probiotic assessment (Hardy et al., 2013). Lactobacilli could affect pro-inflammatory responses [tumor necrosis factor- $\alpha$ (TNF- $\alpha)$, IL- 8 ] and anti-inflammatory responses (IL-10) of eukaryotic cells (HT-29, Caco-2, or other eukaryotic cells) treated with pathogens, lipoprotein, or other factors (Preising et al., 2010; Stöber et al., 2010; Sun et al., 2012). Some Lactobacillus strains can lower secretion of the proinflammatory cytokine IL-8 and promote secretion of the anti-inflammatory IL-10 of intestinal epithelial cells (Butel, 2014; Belguesmia et al., 2016).

Some probiotics have been reported to inhibit pathogenic bacteria by adhering to intestinal epithelium and to decrease pro-inflammatory cytokine expression induced by pathogens (Vanderpool et al., 2008; Liu et al., 2010; Nueno-Palop and Narbad, 2011; Sun et al., 2012; Savino et al., 2015). However, the relationship between inhibiting ability of probiotics against pathogens adhering to epithelial cells and their inhibiting ability against pathogens that induce pro-inflammatory cytokine secretion from epithelial cells is not clear.

In our previous study (Tuo et al., 2013), we studied the adhesion ability of 22 strains of Lactobacillus isolated from traditional fermented dairy food in Nalati, Xinjiqng, China, and some strains with higher adhering ability were considered potential probiotic strains. In this study, 11 Lactobacillus strains with the highest adhesion ability among the 22 Lactobacillus strains were selected to assess their ability to inhibit adhesion of $E$. coli to intestinal epithelial cells and inhibit induction by $E$. coli of IL-8 production by intestinal epithelial cells, and the relationship between the 2 abilities. 
Table 1. Lactobacillus strains evaluated in this study

\begin{tabular}{lll}
\hline Strain & Species & Origin ${ }^{1}$ \\
\hline 22 & Lactobacillus plantarum & Traditional fermented dairy food, Nalati, Xinjiang, China \\
23 & L. plantarum & Traditional fermented dairy food, Nalati, Xinjiang, China \\
39 & Lactobacillus fermentum & Traditional fermented dairy food, Nalati, Xinjiang, China \\
57 & L. plantarum & Traditional fermented dairy food, Nalati, Xinjiang, China \\
66 & L. plantarum & Traditional fermented dairy food, Nalati, Xinjiang, China \\
67 & L. plantarum & Traditional fermented dairy food, Nalati, Xinjiang, China \\
89 & L. plantarum & Traditional fermented dairy food, Nalati, Xinjiang, China \\
130 & L. plantarum & Traditional fermented dairy food, Nalati, Xinjiang, China \\
196 & Lactobacillus casei & Traditional fermented dairy food, Nalati, Xinjiang, China \\
m2.3 & L. casei & Traditional fermented dairy food, Nalati, Xinjiang, China \\
f12 & L. plantarum & Traditional fermented dairy food, Nalati, Xinjiang, China \\
LGG & Lactobacillus rhamnosus GG & Harbin Institute of Technology, Harbin, China \\
\hline
\end{tabular}

${ }^{1}$ The fermented foods were made by local people using traditional methods under local climate and environmental conditions. Traditional fermented dairy foods from different pastures were sampled and strains were isolated from different pastures.

\section{MATERIALS AND METHODS}

\section{Bacterial Strains and Growth Conditions}

Lactobacillus strains tested in this study were isolated from traditional fermented dairy foods in Nalati, Xinjiqng, China, stored in our stock culture collection (Tuo et al., 2013). Detailed information about the strains is listed in Table 1. Escherichia coli ATCC43889 was used from our stock culture collection. Lactobacillus rhamnosus GG (LGG) was a gift from L. Zhang from the Harbin Institute of Technology (Harbin, China). Lactobacilli were cultured anaerobically in de Man, Rogosa, and Sharpe (MRS) broth (Merck KGaA, Darmstadt, Germany) at $37^{\circ} \mathrm{C}$ for $18 \mathrm{~h}$, and E. coli was cultured aerobically in brain-heart infusion (BHI) broth (Difco Laboratories Inc., Detroit, MI) at $37^{\circ} \mathrm{C}$ for $18 \mathrm{~h}$.

\section{Caco-2 and HT-29 Cell Culture}

The human colonic cell line Caco-2 was provided by X. Chen (Ruijin Hospital, Shanghai, China). The Caco2 cells were grown routinely in Dulbecco's modified Eagle medium (DMEM; Gibco/Life Technologies Corp., Grand Island, NY), supplemented with 10\% (vol/vol) heat-inactivated $\left(56^{\circ} \mathrm{C}\right.$ for $\left.30 \mathrm{~min}\right)$ fetal bovine serum (Sijiqing; Zhejiang Tianhang Biological Technology Co. Ltd., Hangzhou, China), penicillin $(100 \mathrm{U} / \mathrm{mL})$, and streptomycin (0.1 g/L; Gibco/Life Technologies Corp.) at $37^{\circ} \mathrm{C}$ in $5 \% \mathrm{CO}_{2}$ in $95 \%$ air. The culture medium was replaced every $48 \mathrm{~h}$ to maintain the cells. Caco- 2 cells were used as intestinal epithelial cell model to study the adhering ability of Lactobacillus strains.

Human colonic cancer cell line HT-29 was obtained from the Chinese Academy of Science (Shanghai, China). The HT-29 cells were cultured in McCoy's medium (Gibco/Life Technologies Corp.) supplemented with $10 \%$ (vol/vol) inactivated $\left(56^{\circ} \mathrm{C}, 30 \mathrm{~min}\right)$ fetal calf serum (Sijiqing Co. Ltd.) in a humidified atmosphere of $5 \% \mathrm{CO}_{2}$ and $95 \%$ air at $37^{\circ} \mathrm{C}$. The HT-29 cells were cultured until they were fully differentiated. The HT-29 cells were used as intestinal cell model to study IL-8 cytokine production induced by $E$. coli.

\section{Effect of Lactobacillus Strains on Adhesion of E. coli to Caco-2 Monolayers}

The effect of Lactobacillus strains on E. coli adhesion was assessed by using Caco-2 cells as an intestinal epithelial cell model according to Ramiah et al. (2008). Caco-2 cells were seeded on 12-well cell culture plates (Greiner Bio-One GmbH, Frickenhausen, Germany) at $1 \times 10^{5}$ cells per well. The plates were cultured at $37^{\circ} \mathrm{C}$ in a humidified atmosphere of $5 \% \mathrm{CO}_{2}$ and $95 \%$ air until a confluent monolayer was obtained.

Caco-2 cell monolayers on the 12-well plates were washed twice with sterile PBS (pH 7.2) before the adhesion assay. Then, 18-h cultures of Lactobacillus strains and E. coli were harvested by centrifugation at 10,000 $\times g$ for 10 min at $4^{\circ} \mathrm{C}$ (Avanti J30I, Beckman Coulter, Brea, CA), washed twice with sterile PBS ( $\mathrm{pH} 7.2$ ), and then resuspended in DMEM (without antibiotic and fetal bovine serum) and adjusted to $1 \times 10^{9} \mathrm{cfu} / \mathrm{mL}$.

To study the ability of Lactobacillus strains to prevent $E$. coli from adhering to Caco- 2 cells by exclusion, $0.5 \mathrm{~mL}$ of each Lactobacillus strain in DMEM suspension was added into wells with Caco- 2 cell monolayers and supplemented with $0.5 \mathrm{~mL}$ of DMEM. After $1 \mathrm{~h}$ of incubation at $37^{\circ} \mathrm{C}, 0.5 \mathrm{~mL}$ of $E$. coli-DMEM suspension was added into the same wells. After $1 \mathrm{~h}$ of incubation, each well of the plates was washed 4 times with PBS (pH 7.2) to remove free, nonattached bacterial cells. Then, $1 \mathrm{~mL}$ of $1 \%$ (vol/vol) Triton X-100 (HFH10, Invitrogen, Carlsbad, CA) was added to each well, and the suspension was stirred to detach the bacterial cells attached to Caco-2 cell monolayers. Serial dilutions of the suspension in sterile PBS were plated 
onto eosin-methylene blue agar (Beijing Aoboxing Biotech Co. Ltd., Beijing, China) and incubated at $37^{\circ} \mathrm{C}$ to determine the $E$. coli bacterial cell number adhering to Caco-2 cells. The inhibiting rate against E. coli adhesion to Caco-2 cells was calculated as follows:

$$
\text { Inhibiting rate }(\%)=\left[1-\left(N_{1} / N_{0}\right)\right] \times 100 \text {, }
$$

where $N_{1}=$ number of $E$. coli adhering to Caco-2 cells and $N_{0}=$ number of $E$. coli added into wells of 12 -well plates initially.

\section{Effect of Lactobacillus Strains on E. coli-Induced IL-8 Production of HT-29 Cells}

The effect of Lactobacillus strains on E. coli-induced IL-8 production by intestinal epithelial cells was assessed by using HT-29 cells as an intestinal epithelial cell model according to Stöber et al. (2010).

The HT-29 cells were seeded in 12 well plates at 2 $\times 10^{5}$ cells/well. The plates were cultured at $37^{\circ} \mathrm{C}$ in a humidified atmosphere of $5 \% \mathrm{CO}_{2}$ and $95 \%$ air for $48 \mathrm{~h}$ before infection. The 18-h cultures of each Lactobacillus strain and $E$. coli were harvested by centrifugation at $10,000 \times g$ for $10 \mathrm{~min}$ at $4^{\circ} \mathrm{C}$, washed twice with PBS ( $\mathrm{pH}$ 7.2), and then resuspended in McCoy's medium (antibiotic-free, fetal bovine serum-free) and adjusted to $1 \times 10^{9} \mathrm{cfu} / \mathrm{mL}$.

We first studied whether the Lactobacillus strains induced IL- 8 production by HT-29 cells. One milliliter of Lactobacillus strain suspension in McCoy's medium (hereafter, Lactobacillus-McCoy's) was added into the wells of a 12-well plate with HT-29 cell monolayers and incubated for $3 \mathrm{~h}$ (study wells); $1.0 \mathrm{~mL}$ of $E$. coli suspension in McCoy's medium (hereafter, E. coli-McCoy's) was inoculated into wells with HT-29 cells and incubated for $3 \mathrm{~h}$ (positive control wells), and McCoy's medium alone was the negative control.

To determine whether the Lactobacillus strains inhibited $E$. coli from inducing IL-8 production by HT-29 cells, $0.5 \mathrm{~mL}$ of suspensions of Lactobacillus-McCoy's and E. coli-McCoy's were added into the wells with HT-29 cell monolayers for $3 \mathrm{~h}$ of incubation. To determine the ability of lactobacilli to inhibit $E$. coliinduced IL- 8 production by HT-29 cells, $0.5 \mathrm{~mL}$ of Lactobacillus-McCoy's was added into wells with HT-29 cell monolayers for $1 \mathrm{~h}$ of incubation, and then $0.5 \mathrm{~mL}$ of E. coli-McCoy's was added into the same wells for 2 $\mathrm{h}$ of incubation. To study the effect of different doses of live and heat-treated Lactobacillus strains on $E$. coli-induced IL- 8 production, $0.5 \mathrm{~mL}$ of live (or heattreated) Lactobacillus-McCoy's (at doses of bacteria: cell $=1,000: 1,100: 1$, and 10:1 respectively) and $0.5 \mathrm{~mL}$ of $E$. coli-McCoy's (at a dose of bacteria:cell $=100: 1$ ) were added into the wells with HT-29 cell monolayers for a 3 -h incubation.

After $3 \mathrm{~h}$ of incubation at $37^{\circ} \mathrm{C}$ in a humidified atmosphere of $5 \% \mathrm{CO}_{2}$ and $95 \%$ air, cell culture supernatants were harvested by centrifugation $(10,000 \times g$ for $10 \mathrm{~min}$ at $4^{\circ} \mathrm{C}$ ) and IL-8 levels were determined by using ELISA kits (Human IL-8 Valukine ELISA kit, R\&D Company, Minneapolis, MN).

\section{Statistical Analysis}

All experiments were performed in triplicate. Statistical treatment of the data was conducted using ANOVA of IBM SPSS Statistics 22 (IBM Corp., Armonk, NY), and Duncan's test was used to compare the means when the overall $P$-value of the experiment was below the value of significance $(P<0.05)$. Mean values and standard errors were calculated and presented as coordinate pairs with error bars.

\section{RESULTS AND DISCUSSION}

\section{Effects of Lactobacillus Strains on Adhesion of E. coli to Caco-2 Cells}

We studied the ability of 11 Lactobacillus strains to prevent E. coli from binding to Caco-2 cell monolayers by exclusion. When the Lactobacillus strains were previously adhered to Caco-2 cell monolayers, adhesion of $E$. coli to Caco-2 cell monolayers was prevented, as shown in Figure 1. Probiotics with epithelial cell binding ability can competitively inhibit pathogen binding, thus hindering their colonization (Yu et al., 2017). The Lactobacillus strains occupied adhering sites on Caco2 cell surfaces, preventing $E$. coli from adhering. Although strains 130 and LGG had lower adhesion ability than other strains, such as strain 57 (Tuo et al., 2013), they had higher inhibiting rates of inhibition against $E$. coli adhering to Caco-2 cell monolayers compared with other strains in this study.

In our previous study (Tuo et al., 2013), the ability of lactobacilli strains to adhere to Caco-2 monolayers was studied. In the current study, the strains with higher adhesion ability did not show higher inhibiting rate against E. coli adhering to Caco-2 monolayers, consistent with the report by $\mathrm{Yu}$ et al. (2017). It was reported that the inhibition of enterotoxigenic E. coli (ETEC) binding to IPEC-1 cells was due to coaggregation (Yu et al., 2017). We found a positive correlation $(P<0.05)$ between the excluding activities of Lactobacillus strains against $E$. coli adhesion and their coaggregation with E. coli, as shown in Figure 2. 


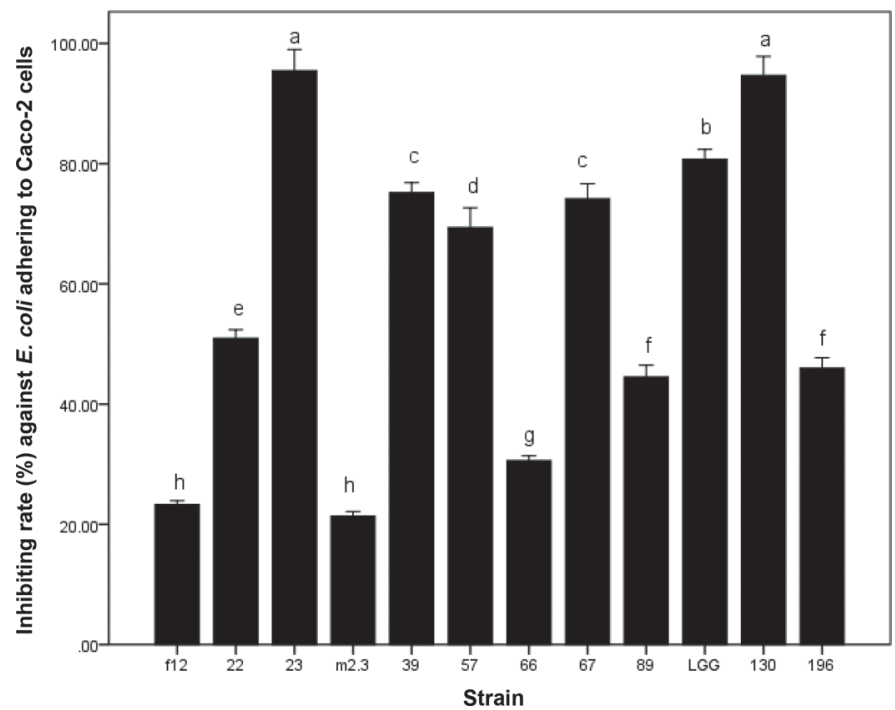

Figure 1. Ability of Lactobacillus strains (see Table 1) to exclude Escherichia coli 0157 from adhering to Caco-2 cells. Different lowercase letters $(\mathrm{a}-\mathrm{h})$ indicate significant differences $(P<0.05)$ among strains. Error bars represent SD.

\section{Effect of Lactobacillus Strains on E. coli-Induced IL-8 Production by HT-29 Cells}

In our previous study (Tuo, 2013), we found that IL-8 production of Caco-2 cells induced by E. coli and Lactobacillus strains was low and not significantly $(P>$ $0.05)$ different from that of Caco-2 cells with no induction, whereas E. coli could dramatically induce IL-8 production in HT-29 cells. Therefore, HT-29 cells were selected to study IL-8 induction in this study.

The effects of different Lactobacillus strains on IL-8 production in HT-29 cells were assessed. As shown in Figure 3, the IL-8 levels of HT-29 cells induced by lactobacilli were significantly lower $(P<0.05)$ than those induced by $E$. coli. The IL-8 levels of HT-29 cells induced by strains 57 and LGG were not different $(P$ $>0.05)$ from those of the negative control, indicating that these strains did not induce production of proinflammatory cytokine IL-8. It has been reported that the Lactobacillus strains not inducing IL-8 production by gastrointestinal epithelial cells could alleviate enteritis (Candela et al., 2008; Panpetch et al., 2016). The chemical difference in cell wall components between probiotic bacteria and pathogenic bacteria may account for the different pro-inflammatory cytokine expression of host cells (Wang et al., 2016).

The inhibiting effect of the Lactobacillus strains against $E$. coli-induced IL-8 production of HT-29 was further studied. As shown in Figure 4, Lactobacillus strains significantly $(P<0.05)$ inhibited $E$. coli induction of IL- 8 production. The results indicated the Lac-

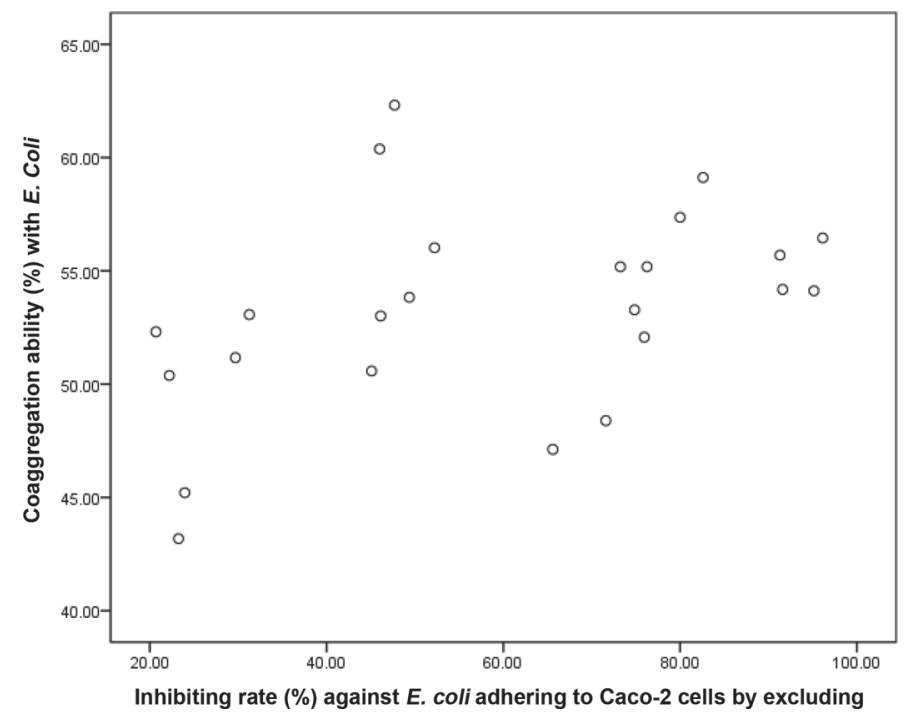

Figure 2. Correlation between inhibiting rates of Lactobacillus strains against Escherichia coli adhesion to Caco-2 cells and their coaggregation with $E$. coli, significant at $P<0.05$.

tobacillus strains could alleviate inflammatory symptoms. It was reported that L. acidophilus Bar13 and Bifidobacterium longum Bar33 alleviated inflammatory symptoms because of lower IL-8 levels in the intestinal tract by displacing the pathogenic bacteria adhering to intestinal epithelium (Candela et al., 2008). In this study, all 11 strains could alleviate E. coli-induced IL-8 production by HT-29 cell monolayers, which could be related to their ability to inhibit E. coli adhesion.

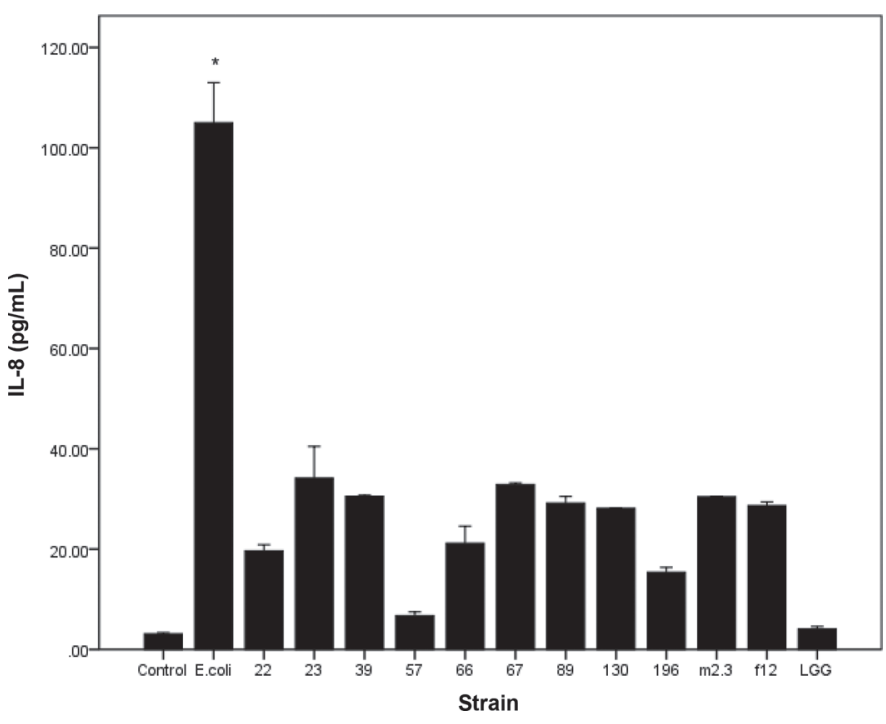

Figure 3. Induction of IL-8 production in HT-29 cells exposed to Lactobacillus strains (see Table 1) or Escherichia coli. *Significant difference $(P<0.05)$ between strains. Error bars represent SD. 


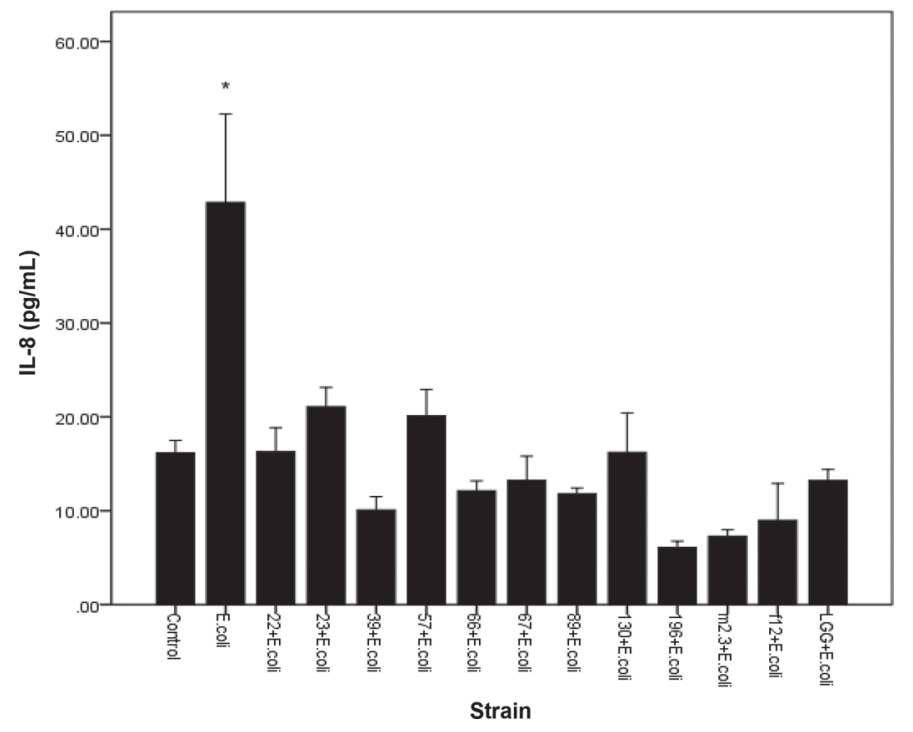

Figure 4. Induction of IL-8 production in HT-29 cells exposed to mixtures of Lactobacillus strains (see Table 1) and Escherichia coli. *Significant difference $(P<0.05)$ between strains. Error bars represent SD.

Seven of the 11 Lactobacillus strains were selected for further study because of their ability to adhere to Caco-2 cells and their inhibitory activity against E.coli-induced IL-8 production by HT-29 cells. When Lactobacillus strain 57, 196, or LGG was cocultured with HT-29 cell monolayers for $1 \mathrm{~h}$ before $E$. coli was inoculated into the mixture, $E$. coli-induced IL- 8 production of HT-29 cells was significantly $(P<0.05)$ inhibited, as shown in Figure 5. Strains 57, 196, and LGG could inhibit $E$. coli-induced IL-8 production by HT-29 cells and inhibit adhesion of E. coli to Caco-2 cells, indicating that these strains had the potential to alleviate intestinal inflammatory symptom induced by pathogens.

\section{Inhibition of Pathogen Adhesion and Pathogen- Induced IL-8 Production}

The inhibiting effect of the Lactobacillus strains against $E$. coli-induced IL-8 production by intestinal epithelial cells may be related to their ability to inhibit adhesion of $E$. coli to intestinal epithelial cells. Bifidobacterium lactis HN019 (Liu et al., 2010) and Lactobacillus crispatus K313 (Sun et al., 2012) were reported to inhibit the attachment of pathogenic bacteria to intestinal epithelial cells and to attenuate pathogen-induced IL-8 expression from intestinal epithelium. In the current study, Lactobacillus strains 57, 196, and LGG exhibited inhibiting activity against $E$. coli, inducing IL-8 production and adhering to Caco-2 cell monolayers. We wanted to determine whether these 2 activities - inhibiting IL-8 production and preventing adhesion - were positively correlated. We did not find a positive relationship between the inhibitory activities of the Lactobacillus strains against E. coli adhesion and against $E$. coli-induced IL-8 production, as shown in Figure 6A, or between the Lactobacillus strains' ability to adhere to Caco-2 cells and their inhibiting activity against $E$. coli-induced IL-8 production, as shown in Figure 6B.

The mechanism for the inhibition by Lactobacillus strains of E. coli-induced IL-8 production should be related to the cell surface-associated molecules. Probiotic functionality has strain specificity depending on the bioactive effector molecules (Bron et al., 2013). Ligands such as flagellin on the surface of pathogenic bacteria promote adhesion and invasion of host by pathogens. However, metabolites of some beneficial bacteria, such as polysaccharide, surface-bound proteins, or peptidoglycan, could protect the host from pathogen invasion, promoting Treg cell function through special ligand-receptor interactions (Belkaid and Hand, 2014). Perhaps special cell wall components and structure of the Lactobacillus strains account for their interaction with host cells. Surface-bound proteins and other macromolecules are known to play a role in the adhering abilities of 22 Lactobacillus strains (Tuo et al., 2013).

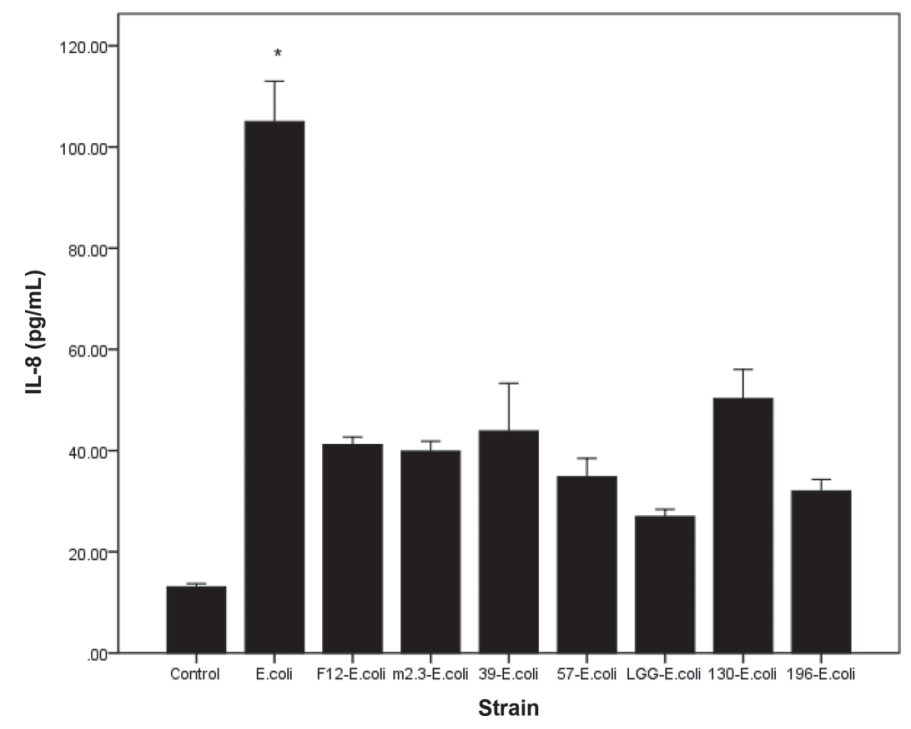

Figure 5. Induction of IL-8 production in HT-29 cells pretreated with Lactobacillus strains (see Table 1) and exposed to Escherichia coli. *Significant difference $(P<0.05)$ between strains. Error bars represent SD. 
A

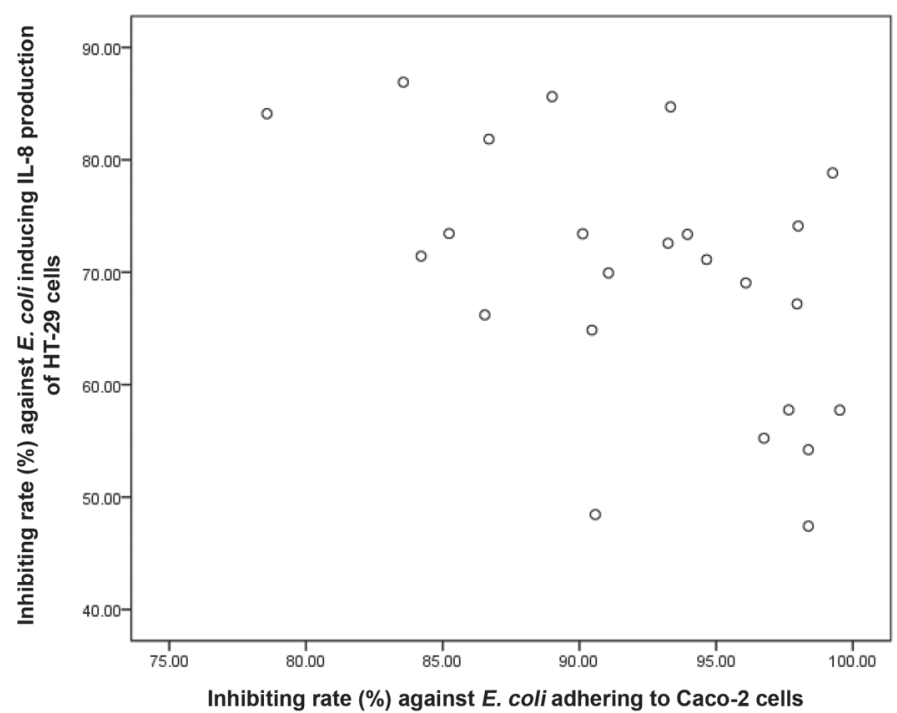

B

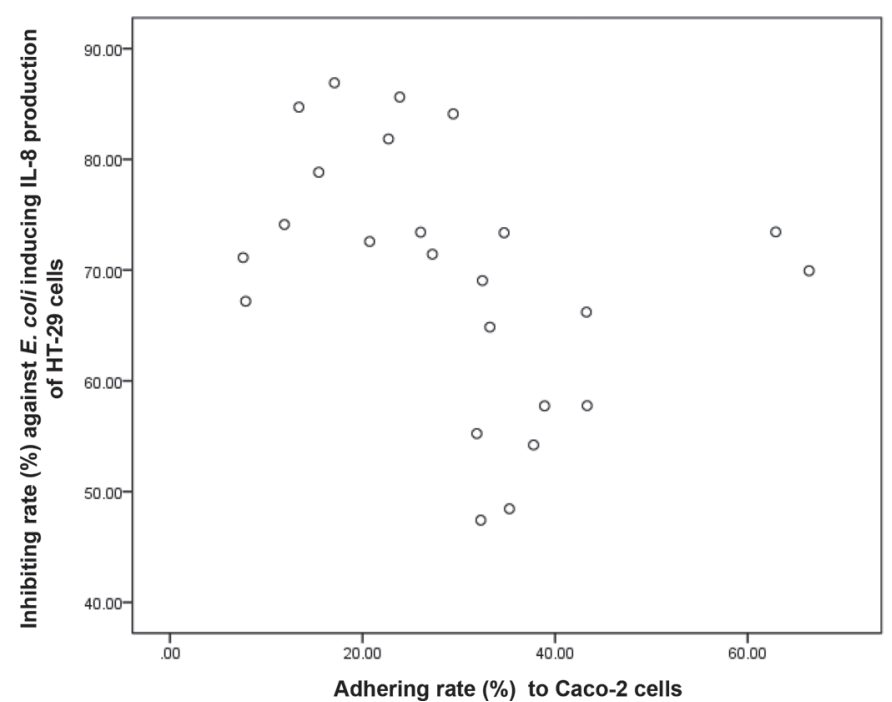

Figure 6. Correlation between adhering ability of Lactobacillus strains (see Table 1) and their inhibiting activity against Escherichia coli invading HT-29 cells. (A) Correlation between inhibiting rates of Lactobacillus strains against E. coli adhesion to Caco-2 cells and inhibiting rates against $E$. coli-induced IL-8 production in HT-29 cells is not significant at $P<0.05$. (B) Correlation between adhering ability of Lactobacillus strains to Caco-2 cells and their inhibiting activity against $E$. coli-induced IL-8 production in HT-29 cells is not significant at $P<0.05$.

\section{Effects of Live and Dead Cells of Lactobacillus Strains on E. coli-Induced IL-8 Production}

We studied the effects of different doses of live and dead bacterial cells of Lactobacillus strains 57, 196, and LGG on $E$. coli -induced IL-8 production by HT29 cell monolayers. The results showed that the IL-8 production decreased with increasing doses of live or dead Lactobacillus strains (Figure 7). In Figure 7A, different doses of live Lactobacillus strains, except strain 57 at a bacteria:cell dose 10:1, could inhibit $E$. coli-induced IL- 8 production of HT-29 cells; in Figure 7B, only heat-killed strains 57 and 196 at a bacteria: cell dose of 1,000:1 significantly $(P<0.05)$ inhibited IL-8 production. Live strains exerted a stronger inhibiting effect against E. coli-induced IL-8 secretion than dead cells. The different effects between live and dead lactobacilli could be due to the different bioactivities of cell surface-associated components. The extracellu-

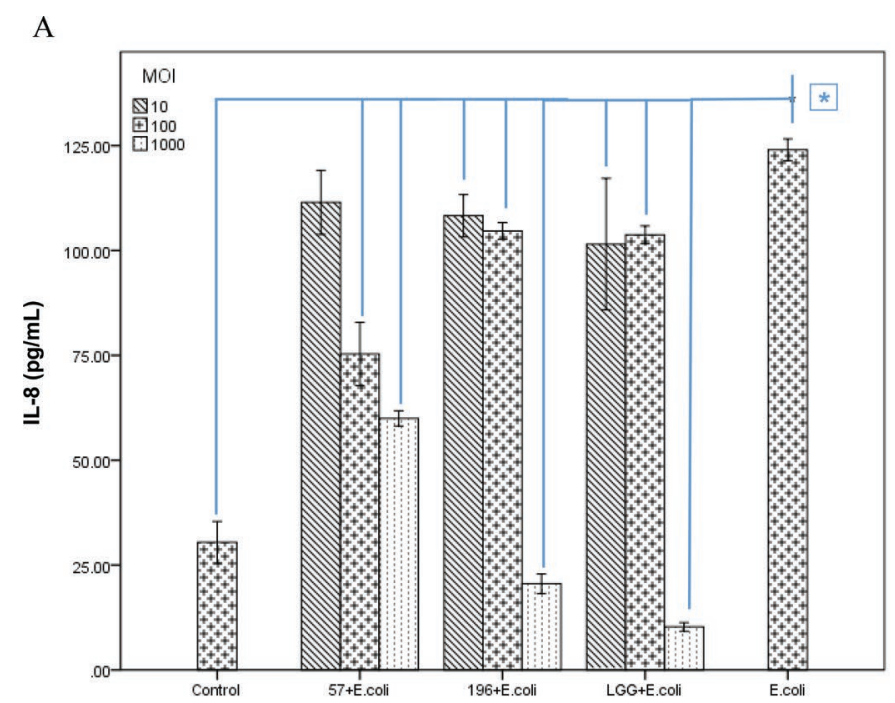

B

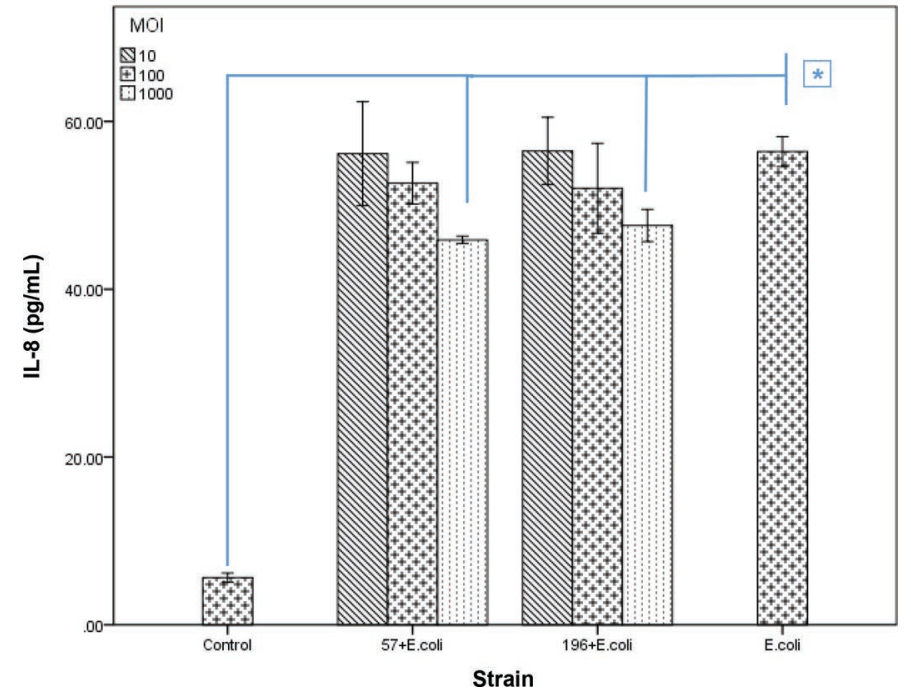

Figure 7. Effect of different doses (bacteria:cell = 1,000:1, 100:1, or 10:1) of Lactobacillus strains (as listed in Table 1) on IL-8 secretion from HT-29 cells induced by Escherichia coli. (A) Live and (B) heattreated Lactobacillus strains' inhibition of E. coli-induced IL-8 production in HT-29 cells. * Significant difference $(P<0.05)$ between bracketed columns. Error bars represent SD. Color version available online. 
lar proteins secreted by Lactobacillus play important roles in preventing adhesion of pathogens to epithelial cells (Sánchez and Urdaci, 2012). It is possible that the heat treatment used in the current study inactivated the bacterial envelope effector molecules, weakening the inhibiting effect of heat-killed bacteria against IL-8 production.

The protective effects of viable lactic acid bacteria against pathogen-induced IL-8 secretion by HT-29 cells were shown to be strain specific, and the protective effects disappeared when viable lactic acid bacteria were disrupted by ultrasound and ball mill (Stöber et al., 2010). Both live and heat-treated cells of Lactobacillus fermentum CECT5716 were shown to attenuate the inflammatory process (Rodríguez-Nogales et al., 2015). Subtle structural differences in the effector molecules expressed by individual lactic acid bacteria are responsible for the strain specificity of probiotic functions (Lee et al., 2013), and structural properties determine the function. In our previous study, the autoaggregating and adhering abilities of 22 Lactobacillus strains decreased because of destruction of the surface-bound proteins by guanidine $\mathrm{HCl}$ (Tuo et al., 2013). In the current study, the bacterial cell structure of the Lactobacillus strains was disrupted by heat treatment, resulting in an alteration or loss of function.

\section{CONCLUSIONS}

In summary, L. plantarum 57 and L. casei 196 had potential to alleviate intestinal inflammatory symptoms induced by pathogens. We did not find a positive relationship between the inhibiting activity of the Lactobacillus strains against E. coli adhesion and their inhibiting activity against E. coli-induced IL-8 production. In this study, inhibiting activities of the Lactobacillus strains against E. coli adhesion and $E$. coli-induced IL- 8 production is strain specific, and the effector molecules of Lactobacillus plantarum 57 and 196 should be identified and purified to explain their effects on $E$. coli adhesion and IL-8 induction.

\section{ACKNOWLEDGMENTS}

This work was financially supported by National Natural Science Foundation Projects of China (Beijing, China; 31571813).

\section{REFERENCES}

Belguesmia, Y., D. Domenger, J. Caron, P. Dhulster, R. Ravallec, D. Drider, and B. Cudennec. 2016. Novel probiotic evidence of lactobacilli on immunomodulation and regulation of satiety hormones release in intestinal cells. J. Funct. Foods 24:276-286.
Belkaid, Y., and T. W. Hand. 2014. Role of the microbiota in immunity and inflammation. Cell 157:121-141.

Bron, P. A., S. Tomita, A. Mercenier, and M. Kleerebezem. 2013. Cell surface-associated compounds of probiotic lactobacilli sustain the strain-specificity dogma. Curr. Opin. Microbiol. 16:262-269.

Butel, M. J. 2014. Probiotics, gut microbiota and health. Med. Mal. Infect. 44:1-8.

Candela, M., F. Perna, P. Carnevali, B. Vitali, R. Ciati, P. Gionchetti, F. Rizzello, M. Campieri, and P. Brigidi. 2008. Interaction of probiotic Lactobacillus and Bifidobacterium strains with human intestinal epithelial cells: Adhesion properties, competition against enteropathogens and modulation of IL-8 production. Int. J. Food Microbiol. 125:286-292.

Chen, Y. P., P. J. Hsiao, W. S. Hong, T. Y. Dai, and M. J. Chen. 2012 Lactobacillus kefiranofaciens M1 isolated from milk kefir grains ameliorates experimental colitis in vitro and in vivo. J. Dairy Sci. 95:63-74.

Collado, M. C., J. Meriluoto, and S. Salminen. 2007. Role of commercial probiotic strains against human pathogen adhesion to intestinal mucus. Lett. Appl. Microbiol. 45:454-460.

Faghfoori, Z., B. P. Gargari, A. S. Gharamalei, H. Bagherpour, and A. Y. Khosroushahi. 2015. Cellular and molecular mechanisms of probiotics effects on colorectal cancer. J. Funct. Foods 18:463-472.

FAO/WHO. 2001. Evaluation of Health and Nutritional Properties of Powder Milk and Live Lactic Acid Bacteria. Expert Consultant Report. 1-34. Food and Agriculture Organization of the United Nations, Rome, Italy, and World Health Organization, Geneva, Switzerland.

Fukuda, S., H. Toh, K. Hase, K. Oshim, Y. Nakanishi, K. Yoshimura T. Tobe, J. M. Clarke, D. L. Topping, T. Suzuki, T. D. Taylor, K. Itoh, J. Kikuchi, H. Morita, M. Hattori, and H. Ohno. 2011. Bifidobacteria can protect from enteropathogenic infection through production of acetate. Nature 469:543-547.

Hardy, H., J. Harris, E. Lyon, J. Beal, and A. D. Foey. 2013. Probiotics, prebiotics and immunomodulation of gut mucosal defences: Homeostasis and immunopathology. Nutrients 5:1869-1912.

Lee, I.-C., S. Tomita, M. Kleerebezem, and P. A. Bron. 2013. The quest for probiotic effector molecules-Unraveling strain specificity at the molecular level. Pharmacol. Res. 69:61-74.

Liu, C., Z. Y. Zhang, K. Dong, and X. K. Guo. 2010. Adhesion and immunomodulatory effects of Bifidobacterium lactis HN019 on intestinal epithelial cells INT-407. World J. Gastroenterol. 16:22832290.

Nueno-Palop, C., and A. Narbad. 2011. Probiotic assessment of Enterococcus faecalis CP58 isolated from human gut. Int. J. Food Microbiol. 145:390-394.

Panpetch, W., J. K. Spinler, J. Versalovic, and S. Tumwasorn. 2016. Characterization of Lactobacillus salivarius strains B37 and B60 capable of inhibiting IL-8 production in Helicobacter pylori-stimulated gastric epithelial cells. BMC Microbiol. 16:242.

Preising, J., D. Philippe, M. Gleinser, H. Wei, S. Buum, B. J. Eikmanns, J. H. Niess, and C. U. Riedel. 2010. Selection of bifidobacteria based on adhesion and anti-inflammatory capacity in vitro for amelioration of murine colitis. Appl. Environ. Microbiol. 76:3048-3051

Ramiah, K., C. A. van Reenen, and L. M. T. C. Dicks. 2008. Surfacebound proteins of Lactobacillus plantarum 423 that contribute to adhesion of Caco-2 cells and their role in competitive exclusion and displacement of Clostridium sporogenes and Enterococcus faecalis. Res. Microbiol. 159:470-475.

Rodríguez-Nogales, A., F. Algieri, T. Vezza, N. Garrido-Mesa, M. Olivares, and M. Comalada. 2015. The viability of Lactobacillus fermentum CECT5716 is not essential to exert intestinal anti-inflammatory properties. Food Funct. 6:1176-1184.

Sánchez, B., and M. C. Urdaci. 2012. Extracellular proteins from Lactobacillus plantarum BMCM12 prevent adhesion of enteropathogens to mucin. Curr. Microbiol. 64:592-596.

Savino, F., S. Fornasero, S. Ceratto, A. De Marco, N. Mandras, J. Roana, V. Tullio, and G. Amisano. 2015. Probiotics and gut health in infants: A preliminary case-control observational study about 
early treatment with Lactobacillus reuteri DSM 17938. Clin. Chim. Acta 451(A):82-87.

Saxami, G., A. Karapetsas, E. Lamprianidou, I. Kotsianidis, A. Chlichlia, C. Tassou, V. Zoumpourlis, and A. Galanis. 2016. Two potential probiotic lactobacillus strains isolated from olive microbiota exhibit adhesion and anti-proliferative effects in cancer cell lines. J. Funct. Foods 24:461-471.

Sánchez, B., and M. C. Urdaci. 2012. Extracellular proteins from Lactobacillus plantarum BMCM12 prevent adhesion of enteropathogens to mucin. Curr. Microbiol. 64:592-596.

Stöber, H., E. Maier, and H. Schmidt. 2010. Protective effects of lactobacilli, bifidobacteria and staphylococci on the infection of cultured HT29 cells with different enterohemorrhagic Escherichia coli serotypes are strain-specific. Int. J. Food Microbiol. 144:133-140.

Sun, Z., L. Huang, J. Kong, S. Hu, X. Zhang, and W. Kong. 2012. In vitro evaluation of Lactobacillus crispatus K313 and K243: High-adhesion activity and anti-inflammatory effect on Salmonella braenderup infected intestinal epithelial cell. Vet. Microbiol. 159:212-220.

Tuo, Y. 2013. In vitro screening of Lactobacillus strains having potential to anti-inflammatory bowel disease and anti-colon cancer. Postdoctoral Research Report. Shanghai Jiaotong Univ., Shanghai.
Tuo, Y., H. Yu, L. Ai, Z. Wu, B. Guo, and W. Chen. 2013. Aggregation and adhesion properties of 22 Lactobacillus strains. J. Dairy Sci. 96:4252-4257.

Unno, T., J. Choi, H. Hur, M. J. Sadowsky, Y. T. Ahn, C. S. Huh, G. B. Kim, and C. J. Cha. 2015. Changes in human gut microbiota influenced by probiotic fermented milk ingestion. J. Dairy Sci. 98:3568-3576.

Vanderpool, C., F. Yan, and D. B. Polk. 2008. Mechanisms of probiotic action: Implications for therapeutic applications in inflammatory bowel diseases. Inflamm. Bowel Dis. 14:1585-1596.

Wang, J., L. Qi, Z. Wu, L. Mei, and H. Wang. 2016. Different effects of lipoteichoic acid from $C$. butyricum and $S$. aureus on inflammatory responses of HT-29 cells. Int. J. Biol. Macromol. 87:481-487.

$\mathrm{Yu}, \mathrm{X}$., J. Koort, A. Lindholm, J. Rintahaka, I. von Ossowski, A. Palva, and U. Hynönen. 2017. A comparative characterization of different host-sourced Lactobacillus ruminis strains and their adhesive, inhibitory, and immunomodulating functions. Front. Microbiol. 8:657. 